\title{
Interactive Individualized Neuroanatomy Labeling for Neuroanatomy Teaching
}

\author{
Felippe T. Angelo \\ Unicamp/FEEC/DCA, \\ Campinas, SP, \\ Brazil \\ trigueiro.angelo \\ @outlook.com
}

\author{
Raphael Voltoline \\ Unicamp/FEEC/DCA, \\ Campinas, SP, Brazil \\ raphavoltoline.rvr \\ @gmail.com
}

\author{
Giuliano R. Gonçalves \\ Unicamp/FCM, \\ Campinas, SP, Brazil \\ giulianoanatomia \\ @gmail.com
}

\author{
Wu, Shin-Ting \\ Unicamp/FEEC/DCA, \\ Campinas, SP, Brazil \\ ting \\ @dca.fee.unicamp.br
}

\begin{abstract}
As the imaging technology and the understanding of neurological disease improve, a solid understanding of neuroanatomy has become increasingly relevant. Neuroanatomy teaching includes the practice of cadaveric dissection and neuroanatomy atlases consisting of images of a brain with its labeled structures. However, the natural interindividual neuroanatomical variability cannot be taken into account. This work addresses the individual gross neuroanatomy atlas that could enrich medical students' experiences with various individual variations in anatomical landmarks and their spatial relationships. We propose to deform the CerebrA cortical atlas into the individual anatomical magnetic resonance imaging data to increase students' opportunity to contact normal neuroanatomical variations in the early stages of studies. Besides, we include interactive queries on the labels/names of neuroanatomical structures from an individual neuroanatomical atlas in a 3D space. An implementation on top of SimpleITK library and VMTK-Neuro software is presented. We generated a series of surface and internal neuroanatomy maps from 16 test volumes to attest to the potential of the proposed technique in brain labeling. For the age group between 10 to 75 , there is evidence that the superficial cortical labeling is accurate with the visual assessment of the degree of concordance between the neuroanatomical and label boundaries.
\end{abstract}

\section{Keywords}

Neuroanatomical Atlas, CerebrA, Mindboggle 101 atlas, MNI-ICBM2009c template, Image-Registration, Brain Labeling.

\section{INTRODUCTION}

Neuroanatomy is considered a problematic and unenviable topic by the medical community. In the early 90s, Jozefowicz [11] observed a great disinterest among the medical students and the development of the "necrophobia syndrome" in approximately half of them. Although most junior neurologists doubt the usefulness of neuroanatomy, its relevance to clinical practice increases as the understanding of neurological diseases, the imaging technology, and the patient-customized treatment improve [3].

Over the past years, teaching neuroanatomy has changed substantially. Several educational technologies [31] have been developed to allow students to experience clinical practices without resorting to tra-

\footnotetext{
Permission to make digital or hard copies of all or part of this work for personal or classroom use is granted without fee provided that copies are not made or distributed for profit or commercial advantage and that copies bear this notice and the full citation on the first page. To copy otherwise, or republish, to post on servers or to redistribute to lists, requires prior specific permission and/or a fee.
}

ditional approaches, such as cadaveric dissection [17] and atlas of neuroanatomy [3]. Despite providing students with a detailed view of brain structure, cadaveric dissection faces the logistics and regulation related to cadaver purchase, storage, and transport [17]. The atlas with photographic images is still the most common way to study brain anatomy at the student's own pace. However, they cannot fully convey the structures' spatial relationships, which is recognized as fundamental to minimally invasive neurosurgery [30]. Besides, both techniques allow exploring only one or a few single brains.

There are many normal variations in a reference landmark's individual locations, either due to age [1] or due to variation in the cortical folds [13]. Fig. 1 illustrates the variations in the postcentral gyrus, highlighted in blue, across the brain of 3 healthy subjects. The Mindboggle individually manually labeled 101 human brain images were yet an attempt to establish normative morphometric variations in a healthy population [13]. Recently, Manera et al. introduced in [20] the Cerebrum Atlas (CerebrA), which is based on an accurate nonlinear registration of cortical (the outer layer) and subcortical (underneath the cerebral cortex) labeling from 
Mindboggle 101 to the symmetric MNI-ICBM2009c template [8], followed by manual editing.

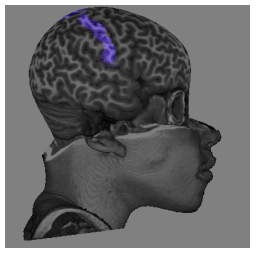

(a) 10-year-old male

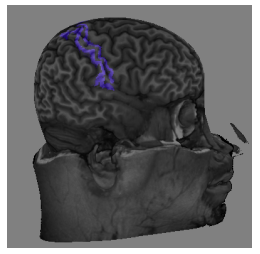

(b) 23-year-old male

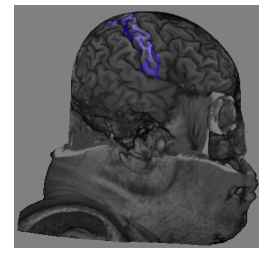

(c) 62-year-old male
Figure 1: Normal variations of a postcentral gyrus.

In this paper, we propose to evaluate the potential of CerebrA in automatic brain labeling of a wide age range for generating a variety of labeled brains of normal variations. We implemented a labeling module and integrated it into the VMTK-Neuro software. VMTK-Neuro provides functions for 3D interactive visualization of neuroanatomical data from the cortical view. Thereby, it helps teachers assess neuroanatomical boundaries in a cortical view and prepare instructional material. Besides, students can explore from these cortical views the normal variations across the available brains.

Our paper is organized as follows. In Section 2, we present an overview of some works that address the exploration and the labeling of neuroanatomical volumes. Next, in Section 3, we provide a detailed explanation of the proposed environment. Then, in Section 4, we present specific information about the software technologies we used for the implementation. After that, in Section 5, we show our results. Finally, in Section 6, we make some concluding remarks. Note that the anatomical slices throughout this paper are displayed in the radiological convention, i.e., the patient's left on the right of the image.

\section{Contributions}

The contributions of this paper are twofold. First, it presents an individual neuroanatomical labeling procedure using the CerebrA atlas to generate labeled brains that differ in normal neuroanatomical variations. Second, we show how to integrate an interactive environment to let a teacher and a student explore the labeled neuroanatomical structures from the cortical superficial views.

\section{RELATED WORK}

The aging population has significantly impacted the increase of neurodegenerative diseases, whose treatment requires a solid understanding of neuroanatomy. As already mentioned, several works have addressed neuroanatomy teaching approaches and tools to spark more interest among preclinical students. This section focuses on the results related to interactive visualization of neuroanatomical atlas and efforts towards brain labeling.

Johnson and Becker [10] led a group that developed a visualization tool for encephalic structures through neuroimages. Thus, it is possible to visualize both labeled structures of healthy and pathological brains. In turn, John Sundsten [27] devised an atlas using the photos of dissection and the drawings of labeled structures. A disadvantage of using these types of the atlas is that the images are limited to brain slices, requiring a spatial cognitive effort to understand the spatial relationships between the cortical surface structures.

Although Martin and Soliz [21] designed an atlas that includes the cortical surface anatomy, the maps are either brain photographs or drawings. Likewise is the teaching website on Pathology, Neuropathology, and Neuroimaging designed by Queiroz and Paes [5]. The informative data comprises only the photographs of brains and microscope slides.

Ding et al. [6] developed a visual exploration tool for a neuroanatomical atlas. Differently from other works, it addressed neuroanatomy at cellular resolution. The atlas was built from anatomical and diffusion-weighted magnetic resonance imaging. Thus, both the gross anatomy and the anatomy of neural pathways can be queried. However, as the previously mentioned works, it is impossible to visualize the labeled structures in three dimensions. Moreover, brain variability was not a concern.

Christensen et al. [4] presented a flow deformation model to transform a generic digital neuroanatomical atlas into the individual brain's shape. They claimed that the annotations of structure names could facilitate the interpretation and analysis of brain scans. In our work, we particularized the case for the CerebrA atlas. We also devised a way to create an annotated volume based on the MNI-ICBM2009c template and visualize the annotated regions in 3D.

The Talairach atlas was created by the neurosurgeons Jean Talairach and Gabor Szikla [28] as a proposal to standardize a grid for the surgery of epilepsy. The grid was based on the conjecture that distances to lesions in the brain are proportional to overall brain size. In 1988 Talairach and Tournoux [29] presented the second version of the Talairach atlas based on post mortem dissection of a 60-year-old woman. Up to now, this atlas has served as the ad hoc standard for reporting locations of activation foci in functional brain mapping studies.

Lancaster et al. [18] further proposed an approach to creating a volume representing a discretization of the Talairach atlas. It allows the registration of an anatomical volume onto the Talairach maps. Consequently, it allows each anatomical volume voxel's association to a Talairach label that corresponds to the cerebral cortex region (Brodmann area). 
Klein et al. [15] showed that the automatic and manual labeling agreement could be significantly improved when multiple atlases were used. In their experiments, they used 20 manually labeled brain images as atlases. Then, they applied the Mindboggle software to automatically label each of the brain scans based on multiple manually labeled scans. The drawback of a multiatlas approach was its high computational cost.

In parallel, the Montreal Neurological Institute (MNI) average brains have been evolving [7]. The first template was the MNI305, an average of 305 T1-weighted magnetic resonance imaging (MRI) scans, linearly transformed into Talairach space. The MNI152, an improvement of the MNI305, was created from the average of 152 T1-weighted MRI scans of a normative young adult population with higher resolution and contrast [7]. It was adopted to define standard anatomy by the International Consortium of Brain Mapping (ICBM).

The linear version of the MNI152 data was evolved to a nonlinear one. A nonlinear MNI152 was built through a series of iterations starting from the MNI152 linear template [8]. Individual native MRIs are nonlinearly fitted to the average template computed in the previous iteration at each iteration. In the most recent phase of the MNI project, 6 versions of the MNI152 can be found and they are divided according to its symmetry and spatial resolution. Fig. 2 shows the axial, sagittal, and coronal slices of the MNI152 (symmetric with 1 $\mathrm{mm}$ of spatial resolution) volume.

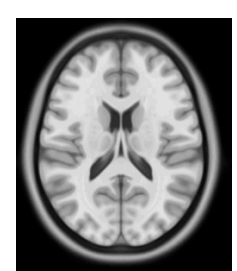

(a) Axial

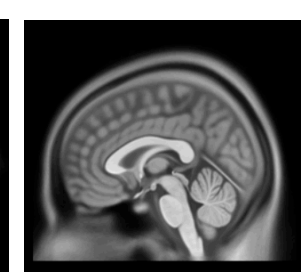

(b) Sagittal

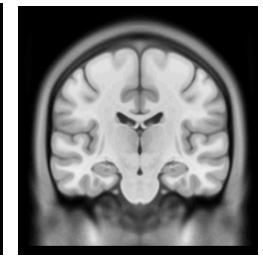

(c) Coronal
Figure 2: MNI152 volume: an average of 152 scans non-linearly transformed to Talairach space.

Finally, in 2009, the MNI released an updated version of nonlinearly registered 152 acquisitions. To date, this template presents the best resolution and detail. Recently, Manera et al. [20] nonlinearly registered the symmetric MNI-ICBM2009 template to the Mindboggle-101 dataset [12], and, after manual editing, they created the Cerebrum Atlas (CerebrA).

\section{OUR PROPOSAL}

In this work, we propose to label a T1-weighted individual volume using CerebrA and allow a teacher to assess the labeling accuracy interactively. We also suggest that students use the same visual assessment environment to explore normal variations in neuroanatomical structures.
Fig. 3 presents an overview of our interactive individual neuroanatomy query tool's architecture comprising two significant modules: labeling and interactive visualization. The labeling module is responsible for estimating a non-linear transformation of the nonlinear symmetric CerebrA [20] into an individual anatomical MRI volume. And the interactive visualization module supports interactive queries of the names and visual feedback of the neuroanatomical structures of an individual MRI volume in both planar and curvilinear reformattings.

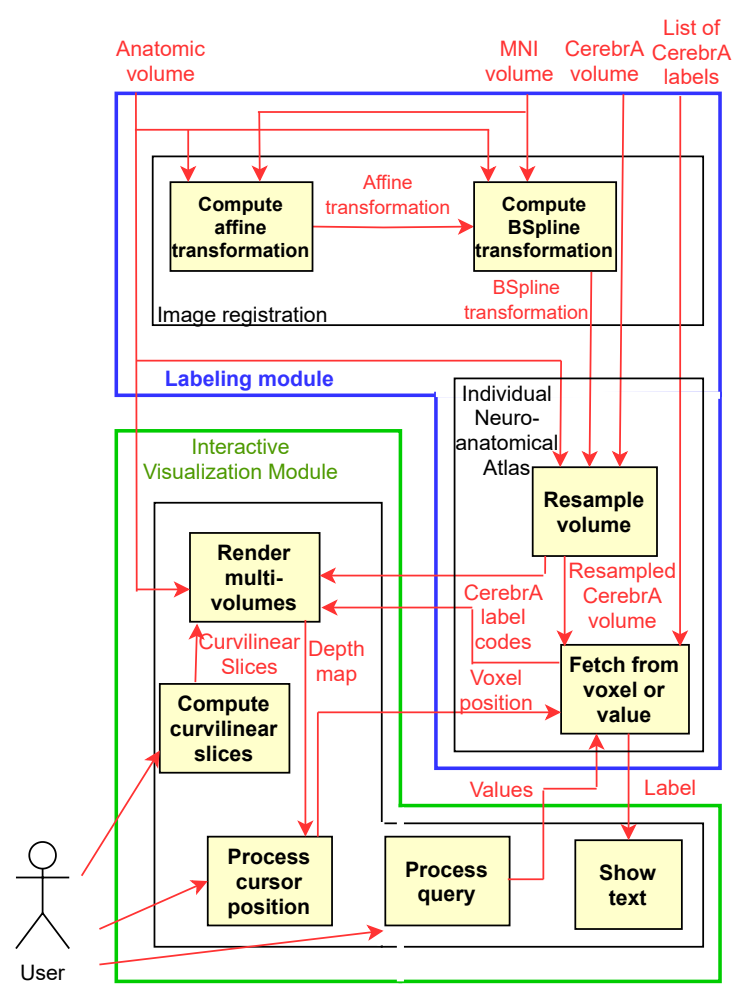

Figure 3: Proposed architecture for an individual interactive neuroanatomy atlas.

\subsection{Labeling Module}

This module, outlined in blue in Fig. 3, is responsible for building an individual neuroanatomy atlas. We proposed to fit the CerebrA into the individual brain. For supporting the voxelwise query of a brain, the CerebrA is resampled in the resolution of an individual MRI volume, as depicted in Fig. 3. We applied the nearestneighbor algorithm to interpolate the labels to ensure the uniqueness of names for each voxel. Then, for the sake of time performance, we performed the registration in two steps.

In the first step, the patient and the MNI152 volumes are coarsely aligned by an affine transformation, as sketched in Fig. 3. Then, the mutual information is the metric applied to evaluate the similarity between two images [22]. This metric is optimized using the Stochastic Gradient Descent algorithm [15]. To improve the algorithm convergence, we opted for a hier- 
archical multiresolution approach [25] with two levels, a level where the images have a quarter of their original size and another level where they have half of their original size.

In the second step, the matching is improved using the Deformable Hierarchical BSpline algorithm [25]. We call it a BSpline transformation, as shown in Fig. 3 because the transformation is based on a grid of BSpline control points that supports the local deformation. We also used the hierarchical multiresolution approach [25] but in this case, with three levels, doubling the number of control points and the size of the images, starting from one-quarter of their original size, from one level to another. In this way, we could deal with coarse differences in lower resolution while adjusting subtle differences in higher resolution. The control points are also calculated by maximizing the mutual information metric [22] using the Stochastic Gradient Descent algorithm.

Fig. 4 illustrates a non-linear registration of the MNI152 symmetric volume (Fig. 4a) to a T1-weighted MRI volume (Fig. 4b) of healthy control. The checkerboard pattern (Fig. 4c) allows assessing the continuity of the neuroanatomical boundaries at the edges of the squares in which the two volumes are shown alternately.

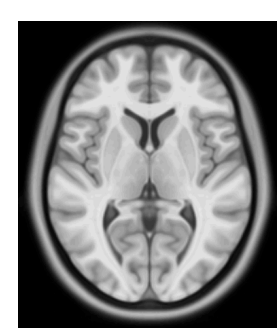

(a) MNI152

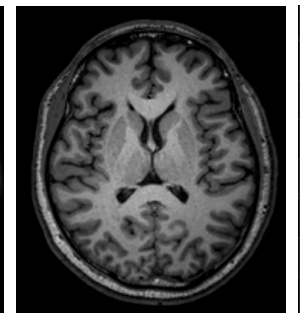

(b) Control Volume

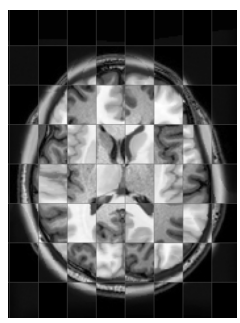

(c) Checkerboard
Figure 4: Non-linear registration of (a) the MNI152 to (b) a T1-weighted volume (c) in a checkerboard pattern.

There is a 1:1 correspondence between the individual MRI voxels and the CerebrA voxels after registration. The voxels in the anatomical MRI volume carry the tissues' response to the applied magnetic field gradient. In contrast, each atlas voxel has one of the 102 label codes available on the NIST website [19]. In short, from a triple of coordinates $(\mathrm{x}, \mathrm{y}, \mathrm{z})$, we could access two kinds of data necessary for rendering: the image of a neuroanatomical structure and its label code.

\subsection{Interactive Visualization Module}

This module, highlighted with the green outline in Fig. 3, is responsible for rendering the MRI volume and the CerebrA atlas into a single image and showing the region of interest's label at interactive rates. Because the registration and the sampling steps are time-consuming but carried out only once for each brain, we considered that they belong to the setup of a session of visual assessment or label queries when the interactions actually occur. For interactive label queries, we should address three issues: the rendering of a labeled anatomical volume at interactive rates, the views of the labeled anatomical MRI volume, and the effective fetch of the structure's name from a point on the cortical surface at which an input device points or the voxels' query from the input structure's name.

\subsubsection{Rendering}

We mapped the structure labels of the CerebrA atlas onto distinct colors according to the FreeSurferColorLUT [14]. We implemented this colormap with a 1D texture lookup table, setting the visible voxel's opacity as 1 and of the other ones 0 . Then, we applied the GPU-based rendering algorithm, proposed by Wu et al.[35], to generate a multimodal volume from the registered CerebrA atlas and a neuroanatomical MRI volume (Fig. 3). It consists of raycasting the anatomical MRI volume. For each pixel in the image, a ray is cast into the volume. The ray is resampled at equal intervals, and the corresponding position in the registered CerebrA atlas is also resampled. The weighted contributions of these two samples are accumulated to define the final color of the pixel. Two interpolation schemes were used in the resampling: the trilinear interpolation to the MRI volume (Fig. 5.(a)) and the nearest-neighbor interpolation to the MNI CerebrA labels (Fig. 5.(b)). Different weighting factors lead to different images, as illustrated in Figs. 5.(c) and (d). Interactive control of the weighting factors facilitates visually checking the alignment between neuroanatomical boundaries and the edges of labeled regions.

\subsubsection{Views}

Besides the classical views of a volume in 3 anatomical planes: axial, coronal, and sagittal (Fig. 6), we devised a way to view the cortical surface at different depths (Fig. 7). It can help one better understanding the spatial relationship between cortical folds and label boundaries. We applied the curvilinear reformatting algorithm presented by Wu et al. [34] to compute curvilinear slices (Fig. 3). The computation consists of four steps: (1) estimating the cortical surface of the brain of interest; (2) computing a mesh of the estimated cortical surface; (3) generating a series of meshes by offsetting the cortical mesh at different depths; and (4) applying the depthmaps of the meshes to control the range of the voxels to be accumulated along the casting ray. A curvilinearly reformatted view of a T1-weighted MRI volume is shown in Fig. 1.

\subsubsection{Interaction}

For querying the label (or name) of any region (show text in Fig. 3), the user interacts with the displayed image through a cursor. Fig. 6 shows a 2D crosshair cursor 


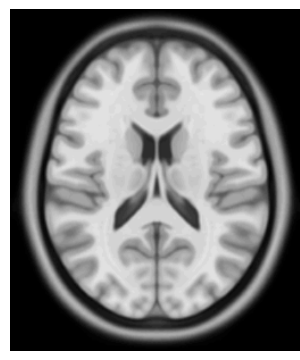

(a) MRI volume

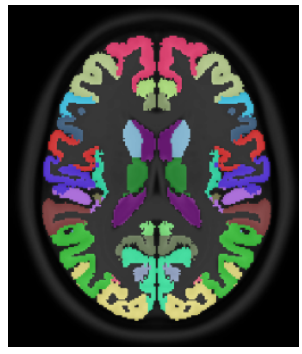

(c) $0.25 \mathrm{MRI}+0.75 \mathrm{MNI}$

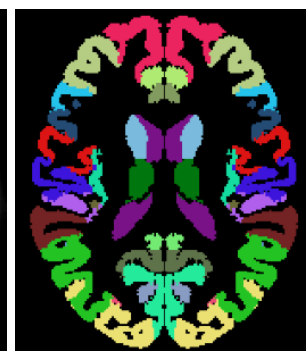

(b) MNI template

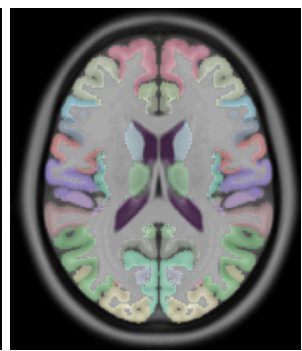

(d) $0.75 \mathrm{MRI}+0.25 \mathrm{MNI}$
Figure 5: Blending of (a) an anatomical MRI volume and (b) the registered CerebrA with (c) $25 \%$ and (d) $75 \%$ of MRI data.

and Fig. 7 a 3D cursor over the right cerebellum on the displayed images as visual feedback of mouse motions. A click with the mouse on a $3 \mathrm{D}$ view of the labeled anatomical volume triggers the procedure that converts the $2 \mathrm{D}$ position of the clicked pixel into the spatial position of the corresponding voxel [33] (process cursor position in Fig. 3). The registered CerebrA label code is accessed from the coordinate $(x, y, z)$ of the picked MRI voxel. We used a balanced binary search algorithm with the worst-case performance $O(\log n)$ to access the CerebrA label code and get the structure's name.

The brain region's query from a structure's name is also supported (process query in Fig. 3). We organized the label codes and their corresponding structure names [14] in a lookup table and applied a linear search to get a label code from a structure name. Though its high cost of $O(n)$, it is affordable due to the small number of structure names. The size $n$ is in the order of magnitude of a hundred. The result could be either in text format (show text in Fig. 3) or in colored regions whose rendering is presented in Section 3.2.1. The two query modes allow the user to explore intuitively and smoothly the neuroanatomical structures individually.

\section{IMPLEMENTATION}

With maximum reuse in mind, we surveyed the applications that provide the functions that would enable the implementation of our proposal. We decided on the functions available in the SimpleITK library [2] to implement the labeling module and the Qt-based
VMTK-Neuro [32] to program the interactive visualization module. SimpleITK is a wrapper for the wellknown ITK [9] and VMTK-Neuro (Visual Manipulation Toolkit for Neuroimages) is a multi-platform (Windows, Mac, and Ubuntu) multi-modal exploratory visualization software. Both are programmed in $\mathrm{C}++$. In addition, built-in functions provided by the algorithm library in C++ standard template libraries (STL) [24] were used to process the CerebrA label codes. With all these tools, we were left with the challenge of integrating them into an application that supports interactive queries of regions covered in the CerebrA atlas.

We distributed the functions between CPU and GPU as depicted in Fig. 8. There is a time-consuming overhead for processing on the CPU an individual CerebrA atlas, which could be saved in a file, as shown in the pink box, and reused in different query sessions. During a query session, the user events are processed by the Qt-based widgets (CPU), and, for the sake of performance, the images are concurrently rendered on the GPU.

We used the functions of the classes IMAGEREGISTRATIONMETHOD, AFFINETRANSFORM and BSPLINETRANFORM, from the SimpleITK image analysis library, to implement the Labeling Module components (Section 3.1). The IMAGEREGISTRATIONMETHOD provides a wide variety of functions that help implement an image registration algorithm. These functions include a series of optimization methods, objective functions, and interfaces to the available classes of transformation models. More specifically, as depicted in Fig. 8, we applied the AFFINETRANSFORM and BSPLINETRANFORM models with, respectively, the parameter values: Maximum number of iterations per level $(200,200)$; Number of hierarchical levels $(2,3)$; Sampling percentages $(10 \%, 10 \%)$; Learning rate $(0.1,0.1)$; Number of histogram bins $(40,40)$; Initial number of control points $(-, 2)$; Convergence value (1e$6,1 \mathrm{e}-6)$; Initial variance of the Gaussian transformation $(4,4)$; Initial shrink factor $(4,4)$; BSpline order $(-, 4)$; Method for rescaling and optimization parameters: (Jacobian, Physical Shift). These values were obtained empirically by registering the 16 control volumes individually.

For benefiting from the interactive environment that the VMTK-Neuro application offers, we have implemented the Interactive Visualization Module on its top. The registered CerebrA atlas and MRI volume should be preloaded as filtered texture into the GPU at the setup of a query session. Two exploration methods are supported: either through 2D anatomical views or through a 3D view of curvilinearly reformatted volume.

A series of curvilinear slices is generated using the functions available in the CURVILINEARREFORMATTING module. The yellow box in Fig. 8 highlights it. We also reused the blending function available in the 


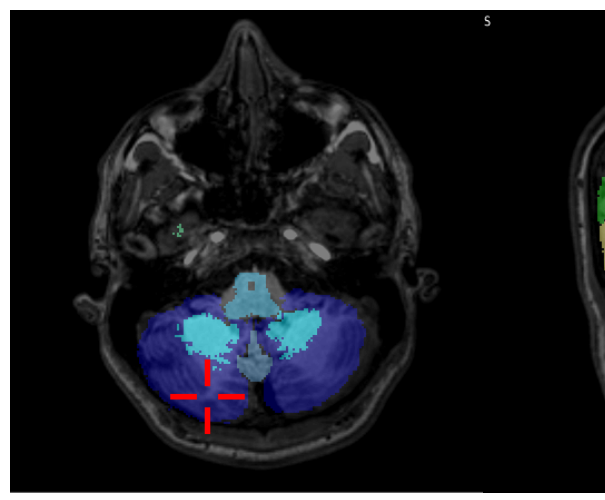

(a) Axial

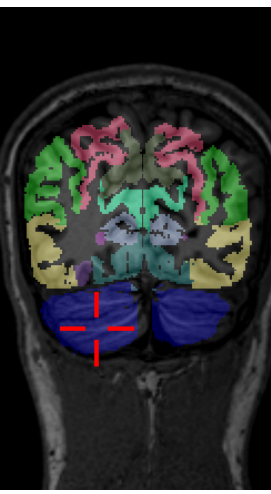

(b) Coronal

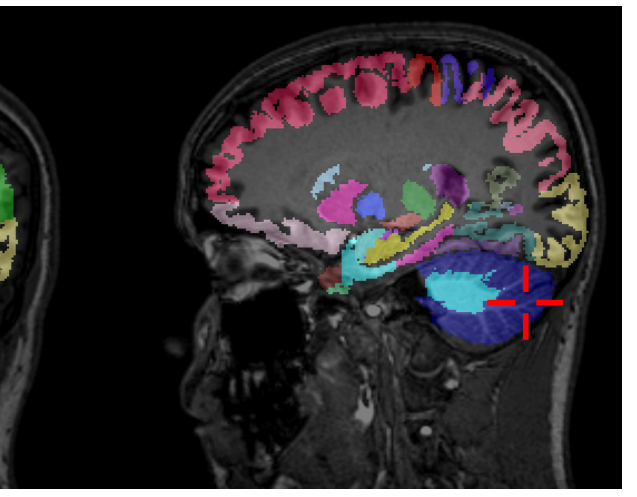

(c) Sagittal

Figure 6: Label querying with a red cursor of the internal structure of neuroanatomy (right cerebellum) from 2D views of 62-year-old male subject.

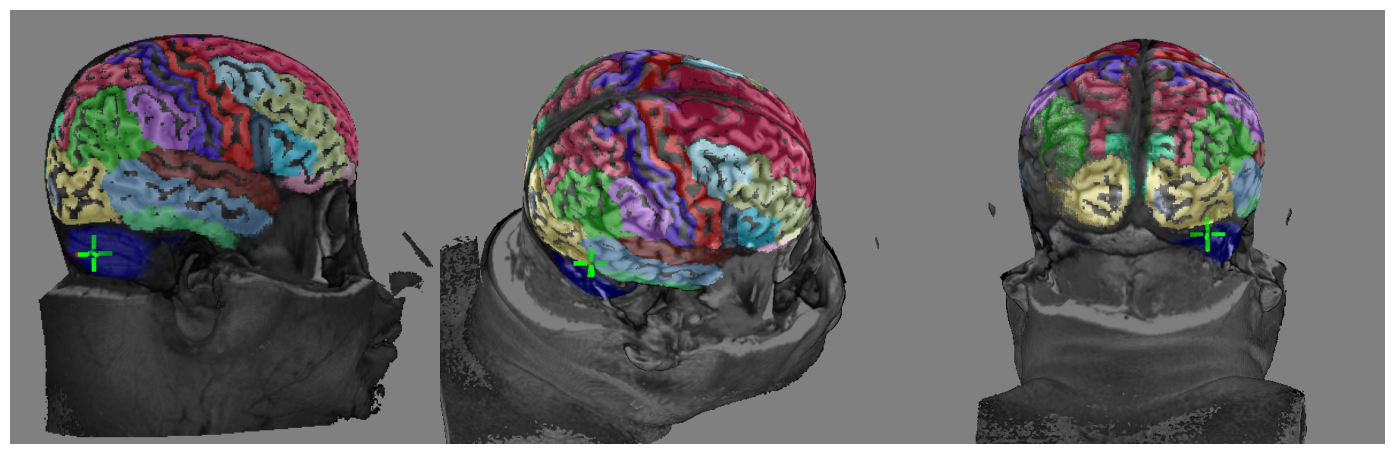

(a) Right

(b) Top

(c) Back

Figure 7: Label querying with a green cursor of surface neuroanatomy (right cerebellum) from 3D views of a 62-year-old male subject.

MulTimODALRENDERING module for weighting the contributions of the anatomical MRI volume and the registered CerebrA atlas to a single rendered image. When not explicitly mentioned, the images presented in this work result from the blending of the label code colors into the grayscale anatomical MRI volume in $50 \%$.

Fig. 6 shows the interface for label queries from the classical anatomical $2 \mathrm{D}$ views. One can retrieve the label by pointing a cursor (a red crosshair) on the colored internal structure in any of three planes (axial, sagittal, and coronal). Fig. 7 illustrates the interface for queries of the (cortical) surface anatomy from a $3 \mathrm{D}$ view of the same subject. The user can retrieve any label by pointing a cursor (a green crosshair) on a colored cortical region.

\section{RESULTS}

Fig. 9 presents the interface of the implemented prototype. It consists of 4 coordinated canvases: one canvas on the left side displays the 3D view of the customized neuroanatomy atlas, and three canvas on the right side displaying axial, sagittal, and coronal slice planes. The cursors in four views point coordinately at the same spatial position. Note that when one clicks a mouse on a point, the name query is automatically triggered. As a result, the value associated with the point's label code is promptly displayed on the widget, outlined by the orange line.

We designed three experiments to show how the proposed environment can visually assess CerebrA-based brain labeling's quality concerning neuroanatomy teaching at interactive rates. First, since accurate registration results in proper labeling, we checked the accuracy of CerebrA's registration to an MRI volume. Second, we visually assessed the concordance between neuroanatomical and label boundaries in all test volumes to rate how misregistration can impact the labeling's visual quality. Finally, in the third experiment, we evaluated the proposed algorithm's time performance for its interactivity.

The experiments were performed on the desktop provided with $8 \mathrm{~Gb}$ of RAM Memory, an Intel Core i7 Processor $8602.8 \mathrm{GHz}$ and an NVIDIA GeForce GT $630 \mathrm{GPU}$, and on the laptop provided with an Intel Core(TM) i7-6700HQ $2.60 \mathrm{GHz}$ with 8GB RAM and an NVIDIA GeForce GTX 960 with 4GB VRAM. We chose randomly from our university hospital's database 16 MRI volumes of healthy subjects ( 8 males) 


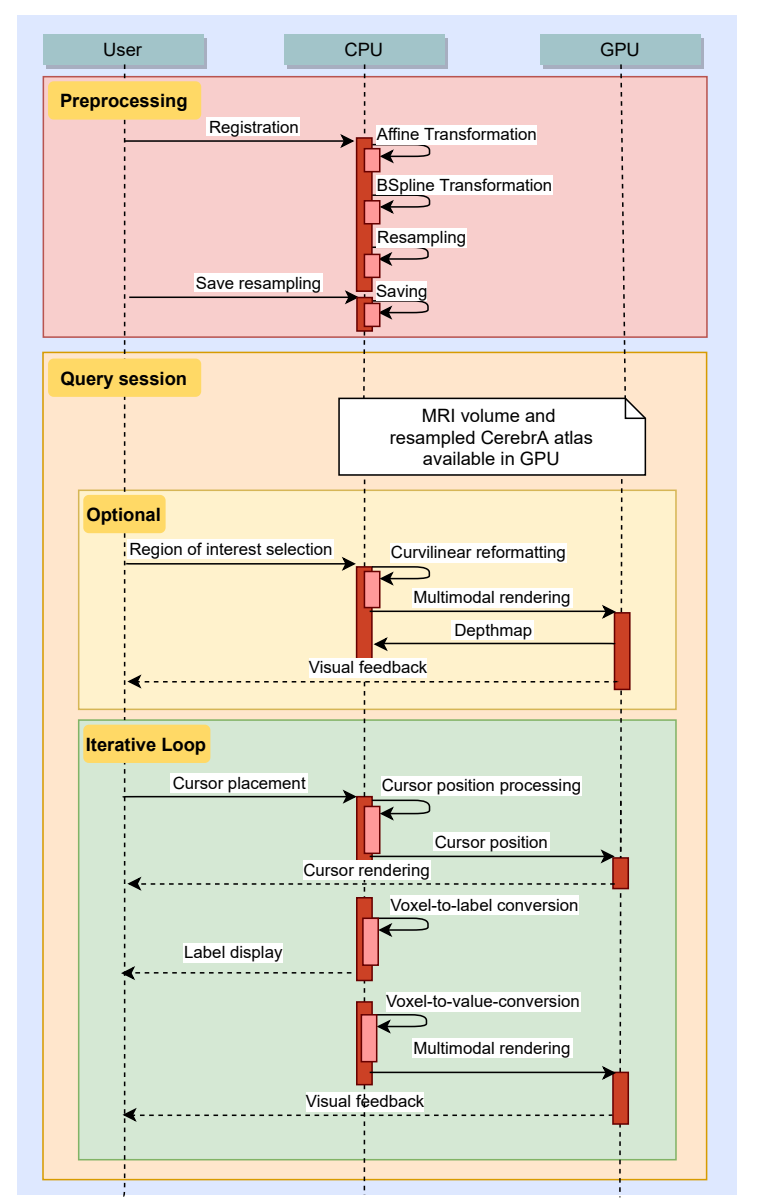

Figure 8: Sequence diagram in two stages: (1) Labeling module: preprocessing of an individual CerebrA atlas using SimpleITK, (2) Interactive Visualization module: query of neuroanatomical structures in the VMTKNeuro environment.

ranged from 9 to 79 years. The T1-weighted spin-echo sequences were acquired on a Philips Achieva 3D Scanner using the acquisition parameters: voxel size $=1 \times 1 \times 1 \mathrm{~mm}$, no gap, TR=7 ms, TE=3.2 ms, flip angle $=8$, matrix $=240 \times 240, \mathrm{FOV}=240 \times 240$, and resolution $=180 \times 240 \times 240$. All subjects enrolled in the present study signed an informed consent form approved by our university's Ethics Committee.

\subsection{Nonlinear Registration}

For assessing the registration accuracy visually, we used the SimpleITK features from Jupyter Notebooks [16]. We applied the HistogRAMMATCHING image filter to the MRI scans before displaying them on a checkerboard pattern, pairwise with the registered MNI152, as shown in Fig. 4. Since the filter standardizes the grayscale values of a control volume by matching the control volume histogram's shape to the MNI152 histogram, it ensures that similar intensities in two volumes have similar tissue meaning [23].
We scrolled the slices in axial, coronal, and sagittal views and checked the continuity of the neuroanatomical boundaries at the squares' edges in each slice. We found that nonlinear registration using the parameters in Section 4 failed for volumes under 30 and over 70 years. Fig. 10a and Fig. 10b illustrate misregistration where there are several mismatches of the neuroanatomical contours along the squares' edges in axial slices. In Fig. 10c, the anatomical boundaries are better matched.

Our finding corroborates the results obtained by Allen et al. [1]. They concluded that gray matter decreased linearly with age, resulting in a decline of about 9,1$9.8 \%$ between the ages of 30 and 70 and a decline of $11.3-12.3 \%$ by 80 . On the other hand, white matter volume increased until the mid-50s; after then, it declined rapidly. At 70 years, white matter volume was only 5.6-6.4\% less than at 30 years, but by age 80 , a cubic regression model predicted that the decrease would be $21.6-25.0 \%$.

\subsection{Labeling}

We carried out the second and the third experiments using the implemented interactive labeling system (Section 4). The visual assessment is based on the agreement of the neuroanatomical and label boundaries from 4 views: the $3 \mathrm{D}$ superficial cortical view, the coronal and axial plane views at the level of septum pellucidum, and the mid-sagittal plane view.

For the first view, we used as anatomical references the frontal, parietal, occipital, and temporal gyri, the lateral and central sulcus, and the longitudinal fissure (LF). For the second view, we chose the LF, the cingulate gyrus (CG), the lateral ventricles (LV), the third ventricle $(3 \mathrm{~V})$, the septum pellucidum (SP), the basal ganglia (BG), the amygdala, the insular cortex (I), and the lateral sulcus. For the third view, the neuroanatomical landmarks were the LF, LV, $3 \mathrm{~V}, \mathrm{SP}$, I, and the corpus callosum (CC). Finally, for the fourth view, the references were the $\mathrm{CG}, \mathrm{LV}, \mathrm{CC}$, cerebellum, the fourth ventricle, the parieto-occipital sulcus, the calcarine sulcus, the brainstem, and the occipital, parietal, and frontal lobes.

As expected, the degree of concordance between the neuroanatomical and label boundaries was low for misregistered pairs of volumes. Fig. 11 illustrates the labeled anatomical structures in the axial slice at the height of the septum pellucidum of the volumes shown in Fig. 10. From the landmarks pointed by the red arrows, one can visually perceive the two boundaries' discrepancies. Our experiment lets us infer that, from the classical plane views, the less the MRI volume is aligned to the CerebrA (Figs. 10a and 10b), the more discrepant are the label boundaries to the anatomical boundaries (Figs. 11a and 11b). 


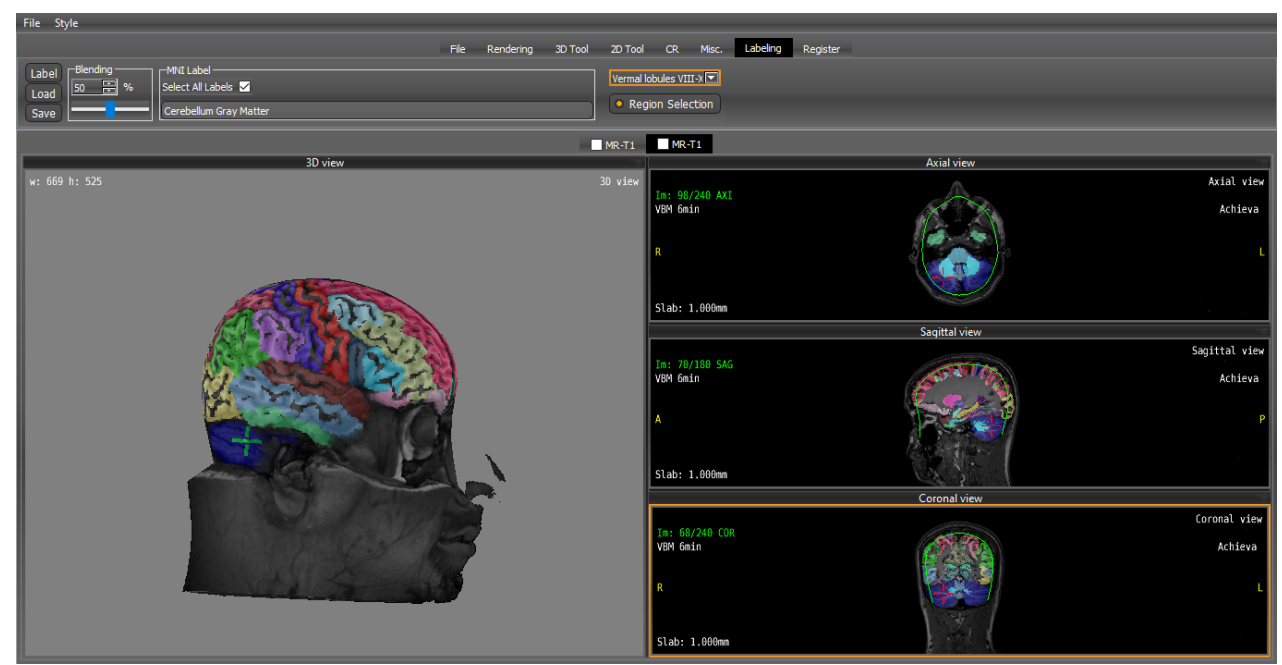

Figure 9: Interface of our proposed individual neuroanatomy atlas. The interface displays the name of the brainstem pointed by the red cursor.

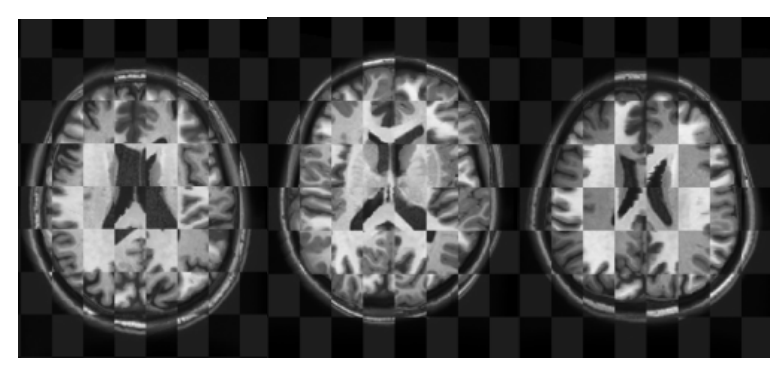

(a) 11 year-old

(b) 78 year-old

(c) 60 year-old
Figure 10: Visual assessment of registration accuracy of a volume: (a) under 30; (b) over 70; and (c) between 30 and 70 years of age.
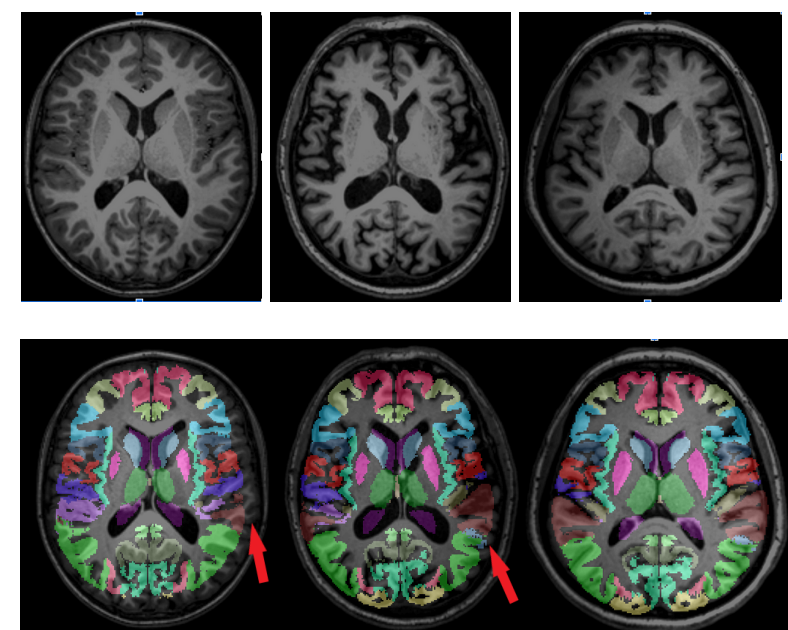

(a) 11 year-old

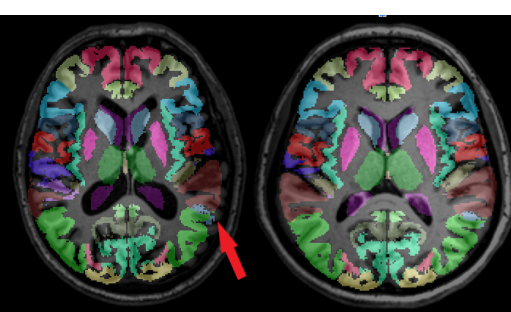

(b) 78 year-old (c) 60 year-old

Figure 11: Visual assessment of labeling accuracy of a volume from axial slices: (a) under 30; (b) over 70; and (c) between 30 and 70 years of age.

However, to our surprise, such discrepancies were not notable from 3D superficial cortical views as depicted in Fig. 12. It looked that the anatomical and label

boundaries were perfectly matchable even when the volumes were misregistered (Figs. 12a and 12b). Only an attentive user would notice some minor flaws in the labeled regions highlighted by the red arrows. Further investigation led us to see that the multimodal rendering procedure we applied accommodates the differences between the superficial boundaries with some slack because it only retrieves the color codes of the control volume's visible voxels. The labels that leaked out the control volume are automatically discarded (Section 3.2.1). The perceived flaws are in the regions where the difference is greater than the cortical thickness, which is few regions.
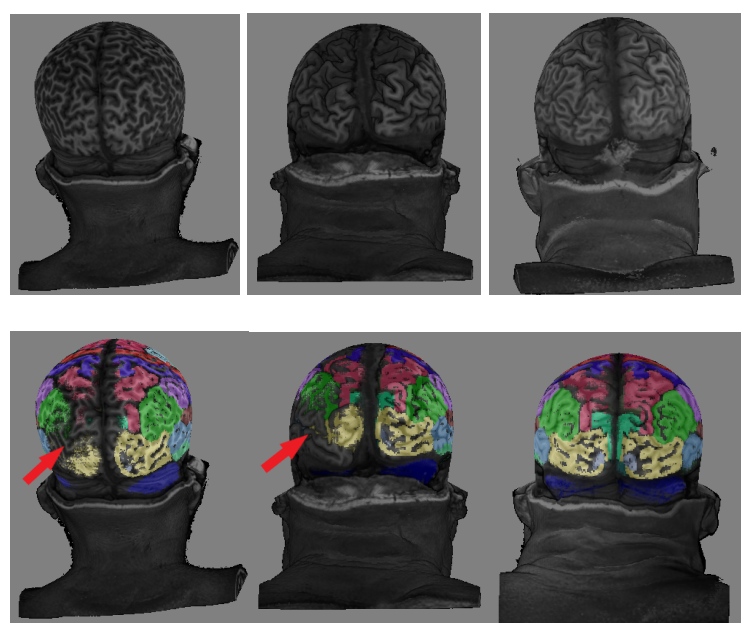

(a) 11 year-old

(b) 78 year-old

(c) 60 year-old

Figure 12: Visual assessment of labeling accuracy of a volume from 3D superficial cortical view: (a) under 30; (b) over 70; and (c) between 30 and 70 years of age.

\subsection{Time Performance}

We measured 3D rendering time and the label query's processing time to evaluate our label querying envi- 
ronment's interactivity. Both measurements were performed on an image of 900x900 pixels. The average times were $42.92 \mathrm{~ms}$ for rendering and $2.67 \mathrm{~ms}$ for query by the label code. Query by a structure's name has taken in average $0.0589 \mathrm{~ms}$. All these times are below the recommended response time limits, suggesting that the environment is interactive. It is, however, worth remarking that the generation of an individual CerebrA atlas is too costly in time. It took less than $20 \mathrm{~min}$ in total on the desktop computer, with around $19 \mathrm{~min}$ to register and 7 s to resample.

\section{CONCLUSION}

We presented an automatic individual CerebrA-based neuroanatomical labeling system to support gross neuroanatomy teaching. Because the CerebrA atlas is based on the symmetric MNI-ICBM2009c template, we conjectured that a nonlinear registration could register CerebrA to most healthy MRI volumes within a margin of tolerance that is acceptable for the teaching purpose. We implemented a prototype on top of available tools and libraries, namely the $\mathrm{C}++$ Standard Template Library [24], SimpleITK [2], and VMTK-Neuro [32].

Our experiments with 16 test control volumes ranged from 9 to 79 years show that the proposed system provides interactive queries and visually accurate labeled cortical surfaces even if the CerebrA atlas could not fit perfectly into an MRI volume of interest. Because this cortical superficial view helps in preoperative planning of the entry points [26], we believe that the developed individual neuroanatomy atlas tool could be useful as complementary courseware to neuroanatomy. Furthermore, afforded curvilinear reformatting and interactive querying by values help students gain familiarity with spatial relationships of the cortical superficial structures of diverse sizes and shapes.

There is still a lot of implementation work to be done. In a short time, we plan to conclude the interface to the querying by values, from which the user can change the color codes and the level of brain hierarchy to be displayed. In the medium term, we also like to integrate the Talairach atlas, which is still widely applied for functional brain mapping. Another point that deserves special attention is the fitting of CerebrA to the age group's volumes outside the range of 30 to 70 years. We would like to know whether there is a procedure to perform this registration in the long run. Finally, we intend to conduct tests directly involving preclinical students to assess the proposed atlas's usability.

\section{REFERENCES}

[1] John S. Allen, Joel Bruss, C. Kice Brown, and Hanna Damasio. Normal neuroanatomical variation due to age: The major lobes and a parcellation of the temporal region. Neurobiology of Aging, 26(9):1245-1260, October 2005.

[2] Richard Beare, Bradley Lowekamp, and Ziv Yaniv. Image segmentation, registration and characterization in $\mathrm{r}$ with simpleitk. Journal of statistical software, 86, 2018.

[3] Bernard S. Chang and Zoltán Molnár. Practical neuroanatomy teaching in the 21 st century. Annals of Neurology, 77(6):911-916, May 2015.

[4] G.E. Christensen, M.I. Miller, M.W. Vannier, and U. Grenander. Individualizing neuro-anatomical atlases using a massively parallel computer. Computer, 29(1):32-38, 1996.

[5] Luciano de Souza Queiroz and Rogério Augusto Paes. Teaching site for pathological anatomy, neuropathology and neurimaging, 2006. Accessed on: July, 2020.

[6] Song-Lin Ding, Joshua J Royall, Susan M Sunkin, Lydia Ng, Benjamin AC Facer, Phil Lesnar, Angie Guillozet-Bongaarts, Bergen McMurray, Aaron Szafer, Tim A Dolbeare, et al. Comprehensive cellular-resolution atlas of the adult human brain. Journal of Comparative Neurology, 524(16):3127-3481, 2016.

[7] Alan C Evans, Andrew L Janke, D Louis Collins, and Sylvain Baillet. Brain templates and atlases. Neuroimage, 62(2):911-922, 2012.

[8] Vladimir Fonov, Alan C. Evans, Kelly Botteron, C. Robert Almli, Robert C. McKinstry, and D. Louis Collins. Unbiased average ageappropriate atlases for pediatric studies. NeuroImage, 54(1):313-327, January 2011.

[9] Hans J. Johnson, M. McCormick, L. Ibáñez, and The Insight Software Consortium. The ITK Software Guide. Kitware, Inc., third edition, 2013. In press.

[10] Keith A. Johnson and J. Alex Becker. The whole brain atlas, 1999. Accessed on: July, 2019 (http://www.med.harvard.edu/ AANLIB /).

[11] R. F. Jozefowicz. Neurophobia: The fear of neurology among medical students. Archives of Neurology, 51(4):328-329, April 1994.

[12] Arno Klein, Brett Mensh, Satrajit Ghosh, Jason Tourville, and Joy Hirsch. Mindboggle: automated brain labeling with multiple atlases. $B M C$ medical imaging, 5(1):7, 2005.

[13] Arno Klein and Jason Tourville. 101 labeled brain images and a consistent human cortical labeling protocol. Frontiers in Neuroscience, 6, 2012.

[14] Arno Klein and Jason Tourville. Mindboggle-101 Labels, jul 2015. Accessed on: Feb 2021.

[15] Stefan Klein, Marius Staring, and Josien PW 
Pluim. Evaluation of optimization methods for nonrigid medical image registration using mutual information and b-splines. IEEE transactions on image processing, 16(12):2879-2890, 2007.

[16] Thomas Kluyver, Benjamin Ragan-Kelley, Fernando Pérez, Brian Granger, Matthias Bussonnier, Jonathan Frederic, Kyle Kelley, Jessica Hamrick, Jason Grout, Sylvain Corlay, Paul Ivanov, Damián Avila, Safia Abdalla, and Carol Willing. Jupyter notebooks - a publishing format for reproducible computational workflows. In F. Loizides and B. Schmidt, editors, Positioning and Power in Academic Publishing: Players, Agents and Agendas, pages 87 - 90. IOS Press, 2016.

[17] Sanjay Konakondla, Reginald Fong, and Clemens M Schirmer. Simulation training in neurosurgery: advances in education and practice. Advances in medical education and practice, 8:465, 2017.

[18] J.L. Lancaster, L.H. Rainey, J.L. Summerlin, C.S. Freitas, P.T. Fox, A.C. Evans, A.W. Toga, and J.C. Mazziotta. Automated labeling of the human brain: A preliminary report on the development and evaluation of a forward-transform method. Human Brain Mapping, 5(4):238-242, 1997.

[19] Ana L. Manera, Mahsa Dadar, Vladimir Fonov, and D. Louis Collins. CerebrA atlas. Kitware, Inc., first edition, 2020.

[20] Ana L. Manera, Mahsa Dadar, Vladimir Fonov, and D. Louis Collins. CerebrA, registration and manual label correction of mindboggle-101 atlas for MNI-ICBM152 template. Scientific Data, 7(1), July 2020.

[21] John H. Martin and Ewa Soliz. Interactive neuroanatomy atlas, 2003. Accessed on: July, 2020 (http://www. columbia.edu/itc/hs / medical/neuroanatomy/neuroanat/) .

[22] David Mattes, David R Haynor, Hubert Vesselle, Thomas K Lewellen, and William Eubank. Pet-ct image registration in the chest using free-form deformations. IEEE transactions on medical imaging, 22(1):120-128, 2003.

[23] L.G. Nyul, J.K. Udupa, and Xuan Zhang. New variants of a method of MRI scale standardization. IEEE Transactions on Medical Imaging, 19(2):143-150, 2000.

[24] P.J. Plauger, Meng Lee, David Musser, and Alexander A. Stepanov. C++ Standard Template Library. Prentice Hall PTR, USA, 1st edition, 2000.

[25] D. Rueckert, L.I. Sonoda, C. Hayes, D.L.G. Hill, M.O. Leach, and D.J. Hawkes. Nonrigid registration using free-form deformations: application to breast MR images. IEEE Transactions on Medical
Imaging, 18(8):712-721, 1999.

[26] Reuben R. Shamir, Leo Joskowicz, Luca Antiga, Roberto I. Foroni, and Yigal Shoshan. Trajectory planning method for reduced patient risk in image-guided neurosurgery: concept and preliminary results. In Medical Imaging 2010: Visualization, Image-Guided Procedures, and Modeling. SPIE, March 2010.

[27] John W. Sundsten. 2-d and 3-d views of the brain from cadaver sections, mri scans, and computer reconstructions., 1997. Accessed on: July, 2019 (http://da.si.washington.edu/ da.html).

[28] J. Talairach and G. Szikla. atlas d'anatomie stereotaxique du telencephale. Masson \& Cie, 1967.

[29] J. Talairach and P. Tournoux. Co-planar Stereotaxic Atlas of the Human Brain. Georg Thieme Verlag, 1988.

[30] Tina Vajsbaher, Holger Schultheis, and Nader K Francis. Spatial cognition in minimally invasive surgery: a systematic review. BMC Surgery, 18(1), November 2018.

[31] Matthew C. Welch, Jonathan Yu, M. Benjamin Larkin, Erin K. Graves, and David Mears. A multimedia educational module for teaching early medical neuroanatomy. MedEdPORTAL, 16(1), January 2020.

[32] S. T. Wu, A. C. Valente, L. d. S. Watanabe, C. L. Yasuda, A. C. Coan, and F. Cendes. Pre-alignment for Co-registration in Native Space. In 2014 27th SIBGRAPI Conference on Graphics, Patterns and Images, pages 41-48, Aug 2014.

[33] S.-T. Wu, José Elías Yauri Vidalón, and Lionis de Souza Watanabe. Snapping a cursor on volume data. In 24th SIBGRAPI Conference on Graphics, Patterns and Images, Sibgrapi 2011, Alagoas, Maceió, Brazil, August 28-31, 2011, pages 109116, 2011.

[34] Shin-Ting $\mathrm{Wu}$, Wallace Souza Loos, Dayvid Leonardo de Castro Oliveira, Fernando Cendes, Clarissa L. Yasuda, and Enrico Ghizoni. Interactive patient-customized curvilinear reformatting for improving neurosurgical planning. International Journal of Computer Assisted Radiology and Surgery, 14(5):851-859, October 2018.

[35] Shin Ting Wu, Raphael Voltoline, Wallace Souza Loos, Jose Angel Ivan Rubianes Silva, Lionis de Souza Watanabe, Barbara Amorim, Ana Carolina Coan, Fernando Cendes, and Clarissa L. Yasuda. Toward a multimodal diagnostic exploratory visualization of focal cortical dysplasia. IEEE Computer Graphics and Applications, 38(3):73-89, 2018. 